\title{
Study on Mesoscopic Stuctural Damage and Permeability Evolution of Freeze-Thaw Shale
}

\author{
Jun-guang Wang ( $\nabla$ shenliu_303@163.com ) \\ Liaoning Technical University \\ Zhang-qing Xuan \\ Liaoning Technical University \\ Qiao Jin \\ Liaoning Technical University \\ Wei-ji Sun \\ Liaoning Technical University \\ Bing Liang \\ Liaoning Technical University \\ Qing-rong Yu \\ Liaoning Technical University
}

\section{Research Article}

Keywords: mesoscopic damage, permeability evolution, rock, freezing-thawing (F-T) cycles, freezingthawing cycle experiments, engineering construction, frozen soil areas

Posted Date: September 15th, 2021

DOI: https://doi.org/10.21203/rs.3.rs-877737/v1

License: (c) (i) This work is licensed under a Creative Commons Attribution 4.0 International License.

Read Full License 


\title{
STUDY ON MESOSCOPIC STUCTURAL DAMAGE AND PERMEABILITY EVOLUTION OF FREEZE-THAW
}

\begin{abstract}
SHALE
WANG Jun-guang ${ }^{1}$, XUAN Zhang-qing ${ }^{1}$, JIN Qiao ${ }^{1}$, SUN Wei-ji ${ }^{1}$, LIANG Bing ${ }^{1}$, Yu Qing-rong ${ }^{1}$

To study the mesoscopic damage and permeability evolution of rock under freezing-thawing (F-T) cycles, freezing-thawing cycle experiments were carried out on shale under different F-T temperatures and cycles, and nuclear magnetic resonance (NMR) and permeability experiments were conducted on shale after F-T. On the basis of the experiment, the pores and permeability of the F-T shale are analyzed, and the existing permeability model is modified and improved; Therefore, the mesoscopic damage evolution characteristics and permeability evolution law of the F-T shale are obtained. It is found that with the increase in the number of cycles, the pore structure of the rock samples changes as the pore size expands and the number of pores increases, and the average porosity also increases correspondingly. It is also found that there is a good positive correlation between the increase in shale porosity and the increase in permeability. Therefore, it is believed that the increase in pore size and pore number leads to an increase in porosity, which in turn leads to an increase in permeability. On the basis of the improved SDR permeability model, the spectral area ratio parameters of large pores and fractures in the $T_{2}$ spectrum were added for correction, and the number of the F-T cycles and temperature parameters were introduced to obtain the modified permeability evolution model of F-T shale. Compared with the experimental results, it is found that the modified model has good applicability. The damage law and permeability of shale under different F-T conditions are analyzed from the microscopic point of view, which has important reference significance for engineering construction in frozen soil areas.
\end{abstract}

\section{Introduction}

Under the influence of temperature, rivers, snow and ice in cold region, many geotechnical engineering projects will inevitably undergo a certain period of F-T cycles.From the development of resources to the construction of subways and mine engineering, the influence of frozen rock on engineering safety will be involved. Therefore, experimental studies on the evolution of meso-damage of rock under different freezing conditions are of great significance for the development and optimization of engineering in frozen regions ${ }^{[1 \sim 2]}$. Therefore, the study of the F-T cycles of shale, a widely distributed rock, is not negligible.

Many scholars have conducted much research on rock freezing and melting. The F-T process is a physical process that has a great influence on rock strength ${ }^{[3]}$. M. Takarli and W. Prince ${ }^{[4 \sim 5]}$ studied the granite permeability and $\mathrm{P}$ wave changes under freezing-melting cycles and obtained 
The permeability of dry and wet granite samples and $\mathrm{P}$ wave changes during 200 freeze-melting cycles. Hiroaki Izumiuama ${ }^{[6-8]}$ et al studied the influence of freeze-melting cycles on the porosity of granite, sandstone and other rocks and established a damage extension model of different weathered bedrock types. Coralie Genty and Jerry L, Jensen ${ }^{[9]}$ et al greatly improved the success rate of identifying rock pore types with complex internal structures by splitting $T_{2}$ into three Gaussian components and classifying the pore types using Bayesian methods. Zhu ${ }^{[10]}$ conducted a digital test of the uniaxial compression deformation damage of marble samples and analyzed the evolution laws of microcracks; Li Jielin and Zhu Longyun ${ }^{[11]}$ studied the influence laws of pore water on sandstone pore changes under freeze-melting cycles. Yao Yanbin and Liu ${ }^{[12]}$ applied NMR technology to shale research, and proposed fine quantitative characterization technology for shale porosity. Li Jielin ${ }^{[13 \sim 15]}$ et al studied the influence of the freezing-melting cycles on sandstone dynamic properties and pore structure. Liu Hanwen ${ }^{[16]}$ established a calculation model of pore volume deformation of sandstone under freezing and melting cycles, and obtained the change law of pore volume. Yu Jin and Zhang Xin ${ }^{[17]}$ studied the influence degree of different sandstone pores under the action of water chemistry and freeze-melting cycles. ZHU Linqi ${ }^{[18]}$ and Bai Songtao ${ }^{[19 \sim 21]}$ et al established a new model of obtaining permeability in different ways. Zhai Cheng and Sun Yong ${ }^{[22]}$ studied the evolution of coal porosity and permeability with the number of circulating freezing and melting cycles. Qin Lei ${ }^{[23]}$ found that the SDR model agrees highly with gas permeability.

The influence of rock material by freeze-melting cycles can be attributed to material deterioration caused by periodic or nonperiodic loading of the material ${ }^{[24-26]}$. Previous experimental studies on freeze-melt cycles focus on the influence of freeze-melt cycles on rock materials, while the impact of freezing temperature changes on rock materials is relatively small. In addition, most of the rocks studying the influence of F-T conditions are concentrated in coal, sandstone and granite, and relatively few studies on shale. Therefore, this paper carries out shale NMR and permeability experiments under different freezing temperatures and times of F-T cycles. Based on the pore structure changes and permeability of shale after F-T, we studied the mesoscopic damage and permeability evolution of shale under freezing-thawing cycles conditions to provide a reference for the F-T shale conditions encountered in engineering practice.

\section{Experiments}

Sample. Taking the Haizhou open-pit mine in Fuxin, Liaoning, as the engineering background, shale rock blocks with good integrity were selected, and the rock blocks collected on site were sealed with plastic film. Then processed the rock sample into a $50 \mathrm{~mm} \times 50 \mathrm{~mm} \times 100 \mathrm{~mm}$ standard cube according to the experimental needs and removed the rock sample test parts that did not meet the specification ${ }^{[27]}$. Through ultrasonic testing of the specimens, the upper and lower surfaces of the specimens that meet the experimental requirements are polished with fine sandpaper. The shale specimens that were partially ready after polishing are shown in Figure 1. 


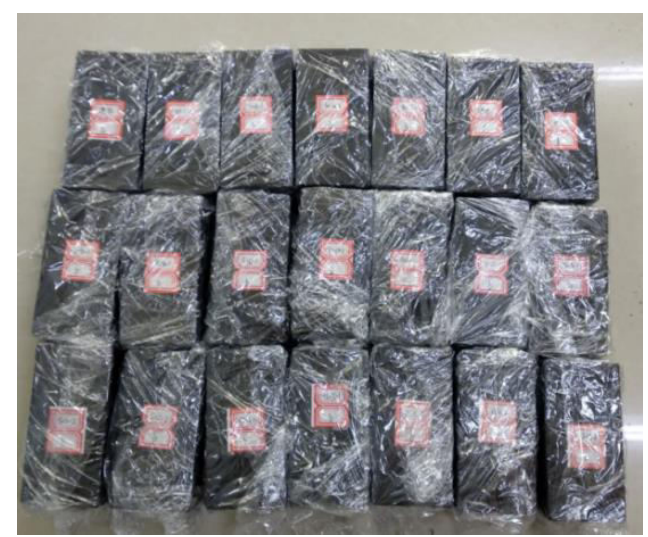

Figure 1 Partial shale samples

The main instrument of the experiment was on MR-60 nuclear magnetic resonance core analyzer form Suzhou Newmai Electronic Technology Co., Ltd. The main magnetic field is $0.51 \mathrm{~T}$, the radio frequency pulse frequency is $1.0 \sim 49.9 \mathrm{MHz}$ and the radio frequency power is $300 \mathrm{~W}$. Main test parameters: the main value of RF signal frequency $\mathrm{SF}$ is $32 \mathrm{MHz}$, magnet temperature $\mathrm{T}$ is $32^{\circ} \mathrm{C}$, single sampling point number TD is 1024 , cumulative sampling number NS is 32 times, echo time TE is $0.233 \mathrm{~ms}$, echo number NECH is 6000 . The permeability experiment adopts an independently developed triaxial permeability instrument, mainly composed of a stress load system and temperature control system, The composition of unified and rock sample holder and data collection system. The experimental equipment also includes a freeze-melt device and ultrasonic detector. The overall principle of the test device is shown in Figure 2.

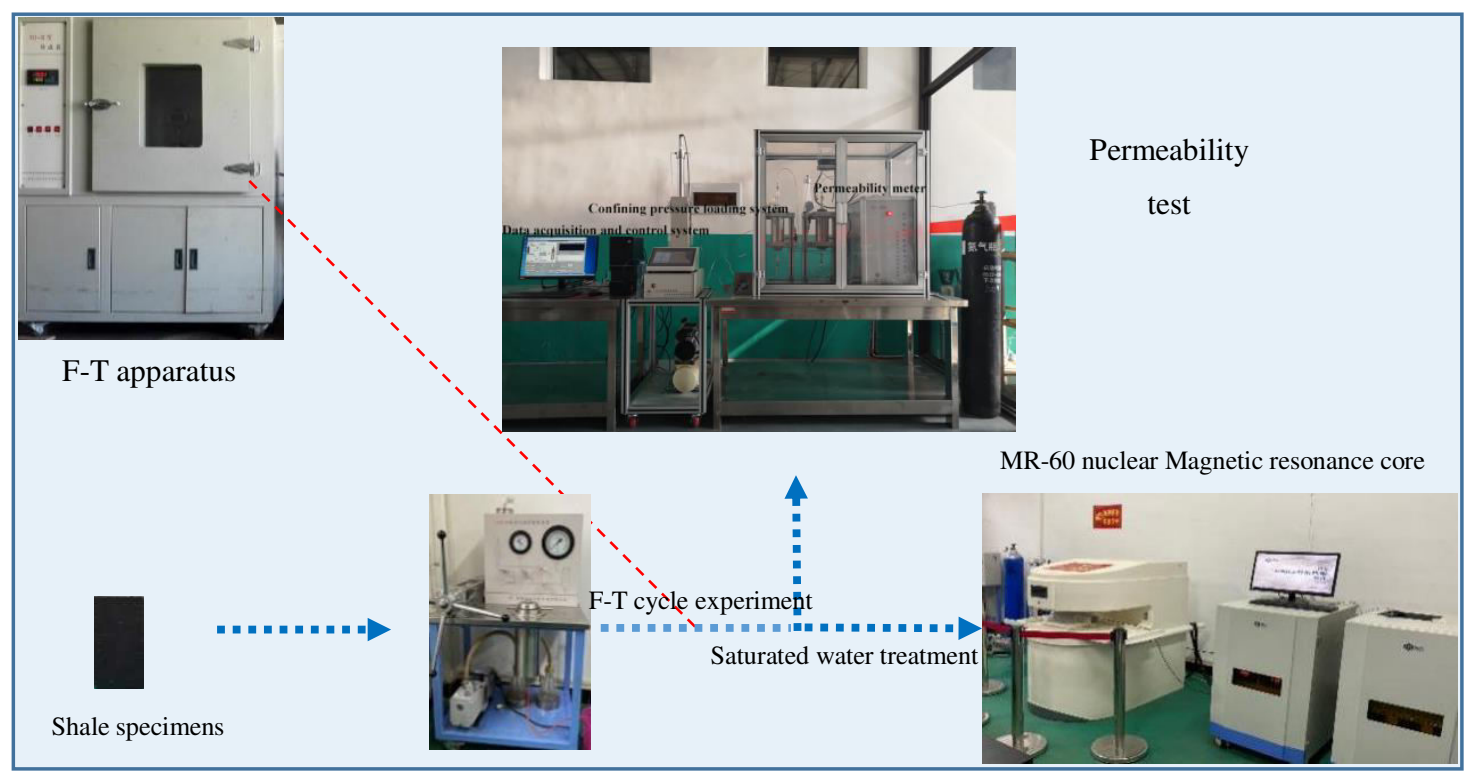

Figure 2 The overall experimental setup

Experimental scheme. The NMR $T_{2}$ spectral distribution curve can represent the rock internal structural information, such as pore size, pore number and pore ratio. The $T_{2}$ value reflects the pore size and the larger the $T_{2}$ value is, the larger the aperture size. The $T_{2}$ spectral peak area is a fixed integral of the $T_{2}$ spectral curve, the area size is proportional to the number of pores, and the area proportion of different peaks reflects the proportion of different pore sizes in the rock mass. The permeability test controls the pressure on the upper and lower surfaces of the specimen through the upper and downstream inlet ends of the pressure chamber. After the specimen is 
saturated, the permeability pressure difference obtained by reducing the upper and lower downstream inlet pressures records the pressure of the rock sample automatically with time and then calculates the permeability of the rock sample.

Before the experiment, the quality and geometric size of the specimen were tested by ultrasonication, and the rock samples with relatively concentrated wave velocities and densities were selected as the experimental rock samples. After the test, 2 rock samples were removed as the control, and the remaining 30 rock samples were divided into 2 groups. The first group of 15 rock samples was tested by NMR after freezing-thawing cycles, and the second group of 15 rock samples was tested by permeability tests after freezing-thawing cycles. The F-T cycle experiments of 30 rock samples were performed under different freezing conditions. The freezing temperature was set at $-5^{\circ} \mathrm{C},-15^{\circ} \mathrm{C}$ and $-30^{\circ} \mathrm{C}$, and the specimen was placed in a constant temperature condition of $20^{\circ} \mathrm{C}$ for $12 \mathrm{~h}$ after freezing for $12 \mathrm{~h}$. This was a freeze-thaw cycle experiment, Before the NMR experiment, the rock sample after the F-T cycle experiment was vacuumized and saturated with a vacuum water saturation device, and the instrument was calibrated with calibration samples. In addition, the calibration method was peak area method. A confining pressure of $5 \mathrm{MPa}$ was set during nuclear magnetic measurement. Nuclear magnetic relaxation was measured in control rock shale with the first set of shale specimens after F-T experiments. NMR $T_{2}$ curves of shale were obtained by inversion of the measurements. We tested the second set of 15 samples after F-T treatment for pulse gas permeability, using helium as the permeation medium, with the surrounding pressure set to $5 \mathrm{MPa}$ and osmolarity set to $2 \mathrm{MPa}$. The pressure of the rock sample surface was recorded by the system over time, thus calculating the permeability of the rock sample.

\section{Results and discussion}

According to the NMR $T_{2}$ spectrum, the shale is mainly composed of micropores and a few mesopores, When the freezing temperature is $-5^{\circ} \mathrm{C}$, there are no large pores or cracks in the whole F-T cycles. However, when the freezing temperature is $-15^{\circ} \mathrm{C}$ and the F-T cycle is 10 times, the freezing temperature is $-30^{\circ} \mathrm{C}$ and the F-T cycle is 5 times, the pores expand and the structures of macropores and cracks begin to appear. The results of porosity and permeability under different freezing temperatures and F-T cycles show that: With the increase of the number of F-T cycles and the decrease of the temperature of F-T cycles, the average porosity and permeability of shale increase, and the influence of the number of F-T cycles on porosity and permeability is more significant.

\section{$T_{2}$ spectral analysis of shale NMR}

The $T_{2}$ spectrum curves measured in the experiment were summarized to obtain the distribution results of the $T_{2}$ spectrum under different F-T times at three freezing temperatures, as shown in Figure 3(a), (b) and (c). Figure 4 shows the proportion of each peak area under different F-T conditions: 


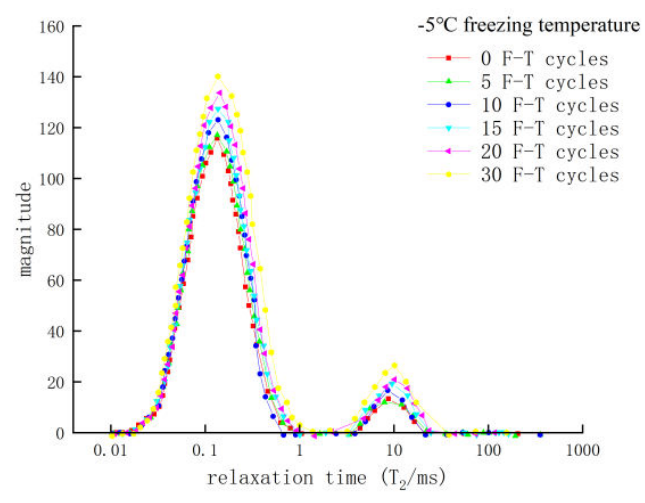

(a) Frozen temperature- $5^{\circ} \mathrm{C}$

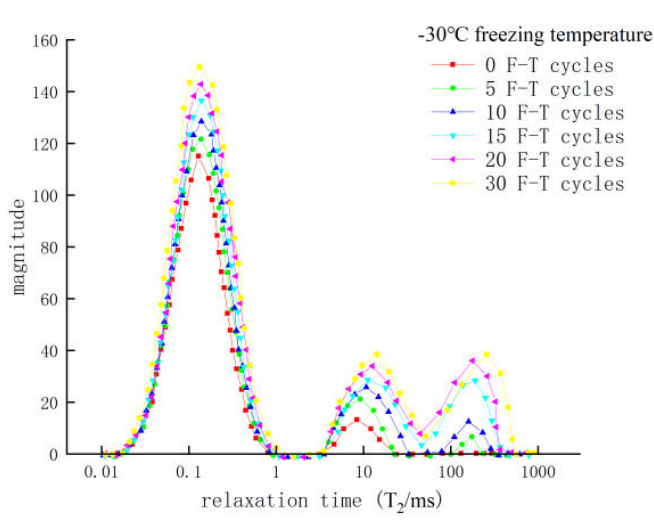

(c) Freeze temperature- $30^{\circ} \mathrm{C}$

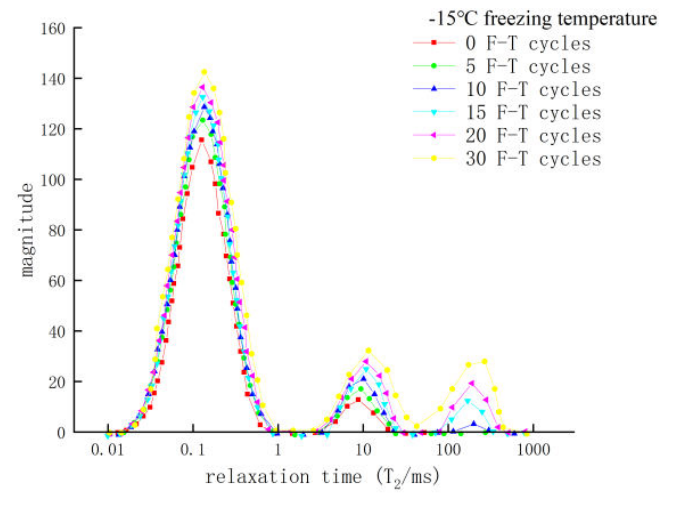

(b) Frozen temperature- $15^{\circ} \mathrm{C}$

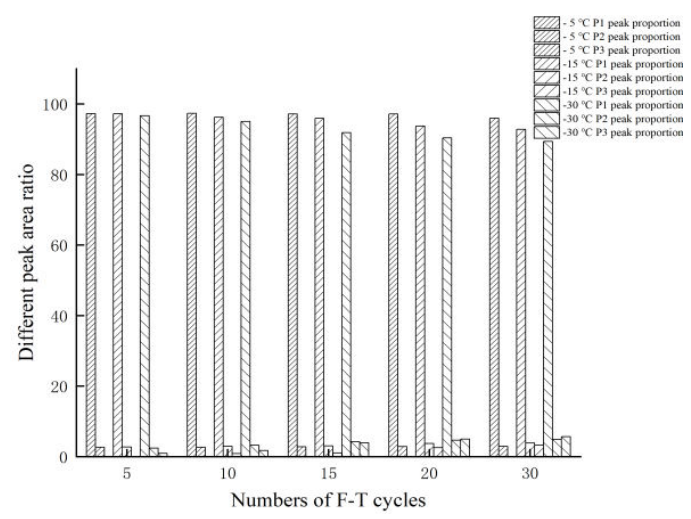

(d) Proportion of peak area under different F-T conditions

Figure $3 \mathrm{~T}_{2}$ Distribution under different F-T conditions

According to the results of Figure 3, when the freezing temperature is $-5^{\circ} \mathrm{C}$, the shale $T_{2}$ spectrum is bimodal. In the whole F-T cycles, the part where the $T_{2}$ value is greater than $100 \mathrm{~ms}$ does not change, and the proportion of peak area between shale specimens does not change much. At this freezing temperature, the deterioration of rock caused by freezing and thawing is very limited. When the number of F-T cycles reached 10, the curve value at a $T_{2}$ value greater than 100 ms gradually rose, The $T_{2}$ curve gradually presented a three-modal morphology. At this time, large holes and crack structures begin to appear. When the freezing temperature is $-30^{\circ} \mathrm{C}$, from the change rule of the $T_{2}$ spectrum, the freeze expansion force generated during the F-T cycle increases significantly in the damage effect on shale samples. Specifically, when the number of F-T cycles is 5, NMR $T_{2}$ spectrum of shale samples shows a three-peak morphology. The peak increase of the three peaks increasing with the number of F-T cycles is more pronounced. Among them, the right peak increased the most significantly during 10 to 15 cycles. The curve overall slightly to the right during 20 to 30 cycles, but the increase slowed. After the F-T cycles, the three peak junctions become sooner and smoother, indicating better connectivity between different pores of different sizes of shale rock samples after the freeze-melt cycles. Through the Figure 3 (d), we learned that the shale NMR $T_{2}$ spectrum has a typical bimodal structure, and the $\mathrm{P}_{1}$ peak value is much higher than the $\mathrm{P}_{2}$ peak value, which is consistent with the initial pore distribution in shale; that is, micropores are dominant, with a few mesopores and no large pores or fractures. When the freezing temperature is $-15^{\circ} \mathrm{C}$, the proportion of the $\mathrm{P}_{1}$ peak area gradually decreases, indicating that some of the micropores have developed into mesopores and macropores. When the freezing 
temperature was $-30^{\circ} \mathrm{C}$, the proportion of $\mathrm{P}_{2}$ and $\mathrm{P}_{3}$ peak areas increased significantly during the whole process, and the $\mathrm{P}_{3}$ peak area changed most significantly. At the same time, there is a small segment with a signal amplitude of 0 at the connection of the two peaks, indicating that the continuity between pores in the shale samples is not good after F-T cycles.

Pore analysis. According to the international porosity classification standard ${ }^{[28]}$, the pore structure was divided into micropores $\left(\mathrm{d}<100 \mathrm{~nm}, T_{2}>2.5 \mathrm{~ms}\right)$, medium pore structures $\left(100 \mathrm{~nm}<\mathrm{d}<1000 \mathrm{~nm}, 2.5 \mathrm{~ms}<T_{2}<100 \mathrm{~ms}\right)$ and large holes and crack structures $(\mathrm{d}>1000 \mathrm{~nm}$, $T_{2}>100 \mathrm{~ms}$ ) according to the range of pore sizes and the corresponding $T_{2}$ values.

To further study the variation in pores of different sizes with the number of cycles at different freezing temperatures, we tried to define the NMR $T_{2}$ spectral area ratio before and after F-T as:

$$
S_{i}=\frac{A_{i_{n}}}{A_{i_{0}}} \quad(i=a 、 b 、 c 、 d)
$$

In the formula, $i=a$ represents the microhole and the small hole, $i=\mathrm{b}$ represents the medium hole, $i=\mathrm{c}$ represents the large hole and the crack, and $i=\mathrm{d}$ represents the total porosity, where $A_{i 0}$ is the $T_{2}$ spectral area of the aperture corresponding to the shale samples treated without freeze-melt cycles. $A_{i n}$ is the $T_{2}$ spectral area of the corresponding aperture under different freezing conditions. Since the different freeze-melting cycle stages of pore structures correspond to $T_{2}>100 \mathrm{~ms}$ under different freezing conditions, the spectral area corresponding to $T_{2}>100 \mathrm{~ms}$ is first observed as $A_{c 0}$ 。

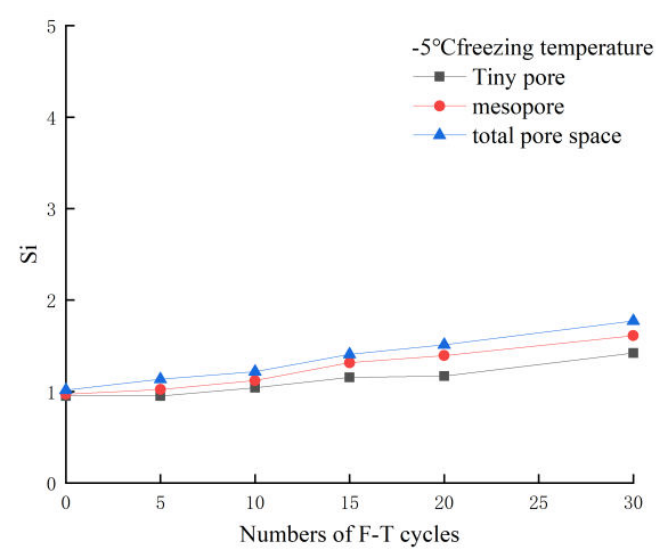

(a)Frozen temperature- $5^{\circ} \mathrm{C}$

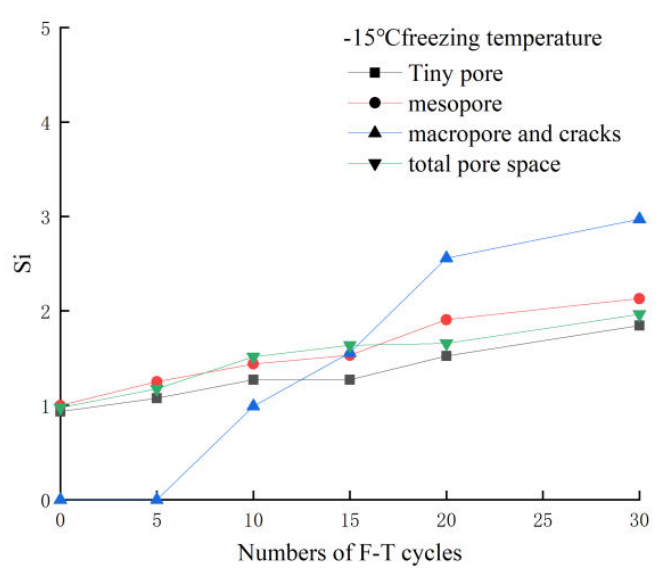

(b) Frozen temperature- $15^{\circ} \mathrm{C}$

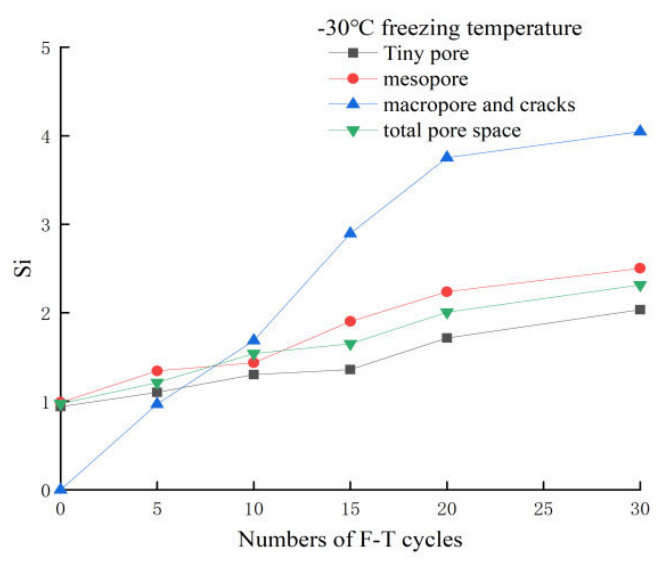


Figure 4 Variation in $\mathrm{Si}$ with the number of F-T cycles at different freezing temperatures

Figure 4 (a), (b) and (c) shows the results of $S_{i}$ at different freezing temperatures to illustrate the distribution rules of pores of different apertures. When the freezing temperature was $-5^{\circ} \mathrm{C}, S_{a-d}$ increased slightly with the number of F-T, which shows that the proportions of microholes, small holes and midholes all increased in the rock samples. The increase in medium pores was higher than that of micropores and pores. The proportion of pores at different apertures changed little before 10 cycles, which shows that the tiny pores still occupy most of the entire pore structure. When the freezing temperature was $-15^{\circ} \mathrm{C}, S_{c}$ appears after reaching 10 cycles, and $S_{c}$ increases significantly during the 10 to $20 \mathrm{~F}-\mathrm{T}$ sessions, which shows that the large pores and cracks develop significantly at this stage, while the increase in tiny pores is limited. The pore structure of shale at this stage changes to small pores developing into medium pores and large holes. Along with the continuous increase in small pores, in the 20 to $30 \mathrm{~F}-\mathrm{T}$ phases, $S_{c}$ growth slowed, the development speed of large pores and cracks is reduced. When the freezing temperature is $-30^{\circ} \mathrm{C}$, the most significant $S_{c}$ change occurs during the 5 to 20 cycles. The $S_{a-c}$ changes before the 15 cycles are relatively slower, which shows that the pores and cracks increase by freezing and melting, while the development of micropores, pores and medium pores is slower. The degree of $S_{a-c}$ change increased after cycles exceeding 15 , small and midholes were continuously generated in rock samples, and the degree of $S_{c}$ changes slowed down after 20 cycles. Large pores and cracks are more strongly affected by freezing and melting than micropores, pores and medium pores, and the affected stage should also be earlier than micropores, pores and medium pores. We found that for small pore apertures, because the early shale internal pore crack network is not developed and there is less water, the expansion effect of the water ice phase is not obvious in the beginning. With the increase in circulation, the pores developed to a certain extent, the degree of water entering the pores gradually increased, and the water ice phase change on its expansion effect gradually became obvious.

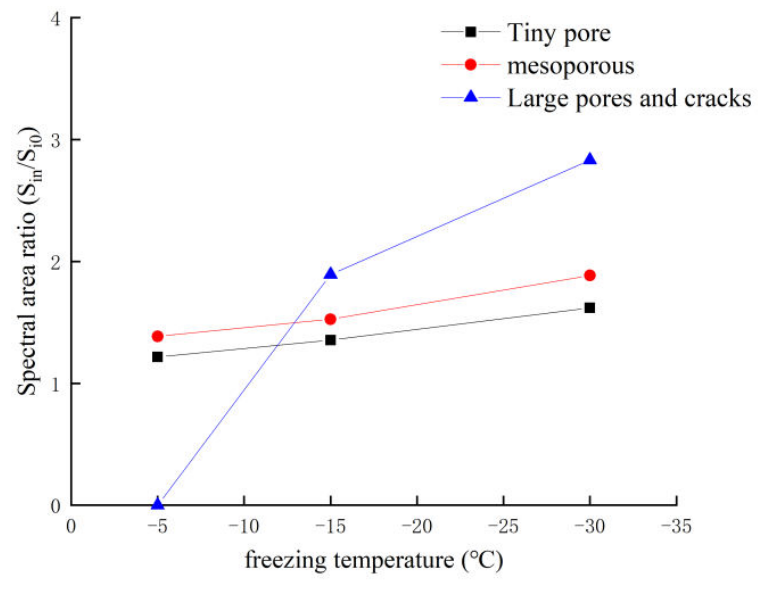

Figure 5 Proportion of pore area of 15 cycles

For the study of the shale pore diameter aperture at the same number of cycles and different freezing temperatures, we determined the proportion of the pore spectral area under 15 F-T cycles. Figure 5 shows that during the 15 F-T cycles, the pores of different aperture sizes all increase as the freezing temperature decreases. Among them, the proportion of the spectral area of micropores and mesopores increased by approximately $9 \%$, and with the decrease in temperature, the 
increasing range also became larger. However, the area proportion of large holes increased by nearly $32 \%$ from $-15^{\circ} \mathrm{C}$ to $-30^{\circ} \mathrm{C}$, and this increase indicates that the large pores and cracks are more affected by temperature.

\section{Analysis of the porosity and permeability}

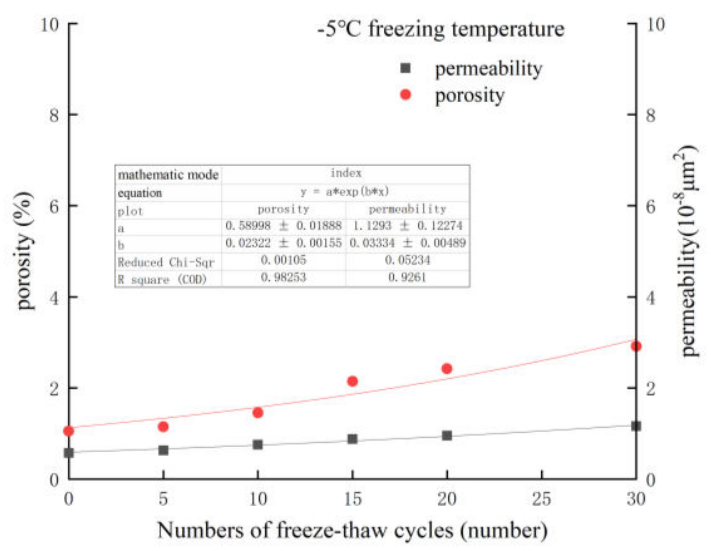

(a) Frozen temperature- $5^{\circ} \mathrm{C}$

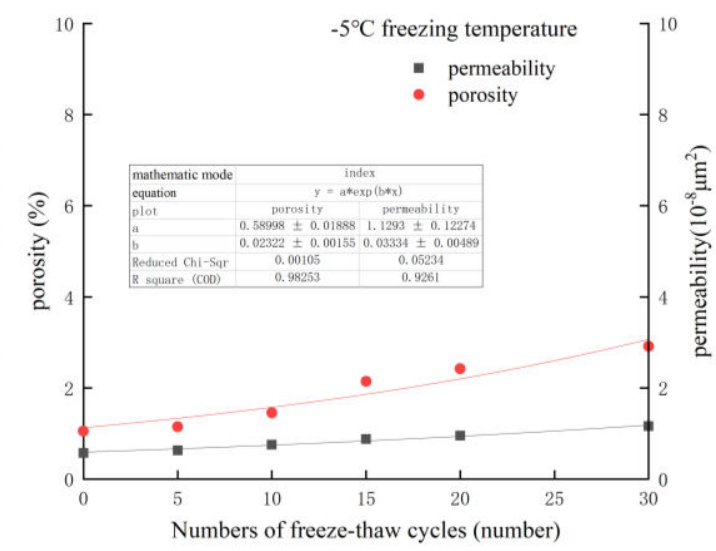

(b)Frozen temperature $-15^{\circ} \mathrm{C}$

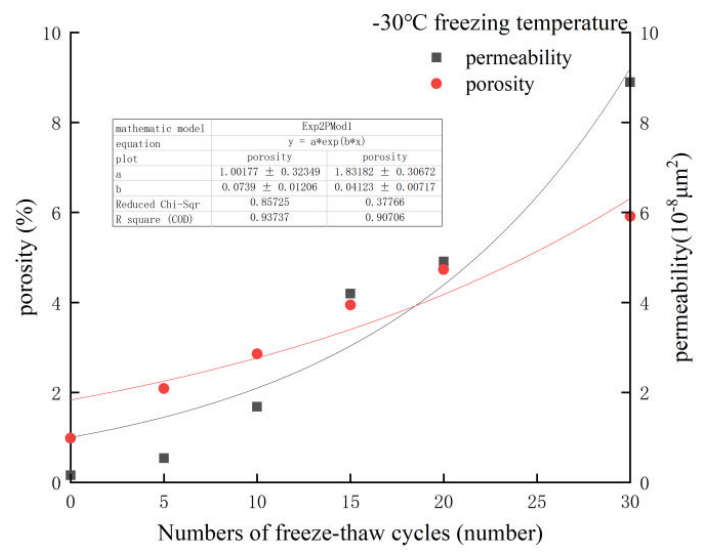

(c)Frozen temperature- $30^{\circ} \mathrm{C}$

Figure 6 Evolution law of shale permeability and porosity with F-T cycles

Figure 6 (a), (b) and (c) show the changes in permeability and nuclear magnetic porosity measured by shale experiments at different freezing temperatures with increasing of F-T times. With the increase in the number of F-T cycles and the decrease in the F-T cycle temperature, the average porosity of shale increases. Through pore size analysis, the spectral area proportion of pores with different pore sizes increased with decreasing of temperature, indicating that pores with different pore sizes became increasingly more abundant and porosity also increased. At the same time, in the process of cyclic F-T, the continuous expansion of micropores, through into mediumand large-sized pores also leads to the increasing porosity of shale ${ }^{[29 \sim 30]}$. Therefore, the change in porosity is considered to be caused by increasing porosity and increasing pore size.

From Figure 6 (a), at the freezing temperature of $-5^{\circ} \mathrm{C}$, there was a small increase in the rock porosity with the number of F-T cycles but no significant change in shale permeability. When the number of F-T cycles reached 30 , the shale permeability was $2.51 \times 10^{-8} \mu \mathrm{m}^{2}$, without an F-T permeability is $0.61 \times 10^{-8} \mu \mathrm{m}^{2}$, which was within the same order of magnitude. According to NMR 
Figure 3 (a), large holes and cracks did not appear during the whole F-T cycle, and the middle hole only changed slightly, so the $T_{2}$ geometric mean change range was small, so the small change in porosity did not significantly improve the permeability of the rock. Figure 6 (b) shows the curve when the freezing temperature was $-15^{\circ} \mathrm{C}$, which shows a more obvious change in the permeability compared with the freezing temperature of $-5^{\circ} \mathrm{C}$. With the increase from the initial $0.61 \times 10^{-8} \mu \mathrm{m}^{2}$ to the final $6.71 \times 10^{-8} \mu \mathrm{m}^{2}$, the permeability was increased by an order of magnitude. The permeability increases the most significantly during 15-30 F-T cycles, which is also the obvious increase in shale porosity. When the freezing temperature is $-30^{\circ} \mathrm{C}$, Figure 6 (c) shows the most significant increase with the number of F-T cycles, and by 30 cycles, the rock permeability reached $35.63 \times 10^{-8} \mu \mathrm{m}^{2}$. An order of magnitude increase in initial permeability began to occur. The significant increase in medium- and large-sized pores during 10-30 F-T cycles indicates that the development of large pores and fissure structures has a greater impact on permeability, and the permeability increases significantly with porosity.

\section{Theoretical model}

Permeability is a measure of how easily fluid flows through rock pores and throats ${ }^{[20]-21]}$. In many permeability models, the improved SDR permeability model is relatively good in calculating rock permeability. The improved SDR permeability model is:

$$
K=a \times \varphi^{b} \times T_{2 g}^{c}
$$

where $\mathrm{K}$ is permeability, $\varphi$ is porosity, a,b,c are model parameters and $T_{2 g}$ is the geometric mean. It is seen above that the influence of large holes and cracks on the permeability is much higher than that of the remaining pores, so the proportion of $S_{c}$ is introduced on the basis of formula (2)

$$
K=a \cdot \varphi^{b} \cdot S_{c} \cdot T_{2 g}^{c}
$$

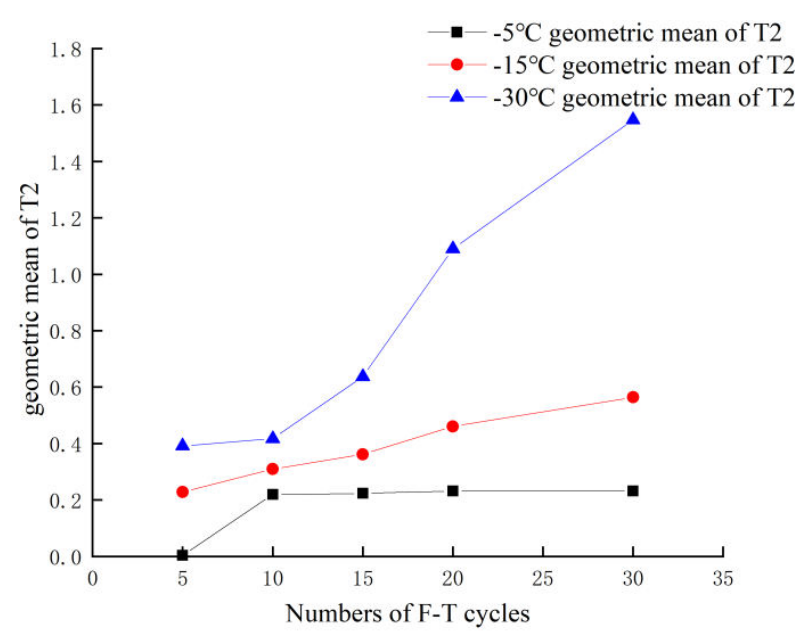

Figure 7 Geometric average of cycles at different freezing temperatures

Table 1 Experimental Measurement of Permeability under Different F-T Cycle Conditions 


\begin{tabular}{cccc}
\hline $\begin{array}{l}\text { Numbers of } \\
\text { F-T cycles } \\
\text { /time }\end{array}$ & $\begin{array}{c}-5^{\circ} \mathrm{C} \\
\text { permeability } \\
\left(10^{-8} \mu \mathrm{m}^{2}\right)\end{array}$ & $\begin{array}{c}-15^{\circ} \mathrm{C} \\
\text { permeability } \\
\left(10^{-8} \mu m^{2}\right)\end{array}$ & $\begin{array}{c}-30^{\circ} \mathrm{C} \\
\text { permeability } \\
\left(10^{-8} \mu m^{2}\right)\end{array}$ \\
\hline 0 & 1.39956 & 1.48722 & 1.18504 \\
5 & 1.52486 & 1.85676 & 3.20107 \\
15 & 1.86057 & 2.06849 & 8.52975 \\
20 & 2.09398 & 3.19139 & 17.76631 \\
25 & 2.40937 & 5.3249 & 20.96449 \\
30 & 2.85505 & 7.02906 & 38.71187 \\
\hline
\end{tabular}

After regression analysis according to the experimental permeability in Table 1 and Figure 7, the parameters $a=4.32 \times 10^{-7}, b=1.0642$ and $c=2.1632$ were obtained. Thus the formula (3) is:

$$
K=4.32 \times 10^{-7} \cdot \varphi^{1.0642} \cdot S_{c} \cdot T_{2 \mathrm{~g}}^{2.1632}
$$

To quantitatively analyze the impact of freezing temperature and circulation number on shale permeability, the circulation number and freezing temperature parameter are introduced on the basis of formula (4) to replace the porosity $\varphi$ in the model parameter.

During F-T cycles, the change in rock sample freezing temperature $\mathrm{T}$ with time $\mathrm{t}$ can be described by the classical heat conduction equation:

$$
C \frac{\partial T}{\partial \mathrm{t}}+\nabla(\lambda \nabla T)=Q_{t}
$$

Where $\mathrm{Q}_{\mathrm{t}}$ is a heat source, $\nabla$ is the Hamiltonian operator and $\mathrm{C}$ is the equivalent volume thermal capacity of saturated rock.

An equivalent thermal parameter $\lambda$ is applied to the control equation. For saturated rock, the equivalent thermal conduction coefficient is expressed by water and solid skeleton particles by volume average:

$$
\lambda=\varphi \lambda_{\mathrm{w}}+(1-\varphi) \lambda_{s}
$$

Where $\varphi$ is porosity, $\lambda_{\mathrm{w}}$ and $\lambda_{\mathrm{s}}$ is thermal conductivity of water and solid skeleton particles respectively; Similarly, the equivalent volume thermal capacity of saturated rock is:

$$
C=\varphi \rho_{\mathrm{w}} C_{w}+(1-\varphi) \rho_{s} C_{s}
$$

Formulas (2) and (3) are substituted into the classical heat conduction equation (1), and the porosity equation is obtained with the fitting equation and different temperatures in Fig. 7 :

$$
\varphi=\mathrm{t}_{1} \cdot \exp ^{t_{2} \cdot x} \quad \mathrm{R}^{2}=0.89
$$

In the formula, $\varphi$ is the porosity, $\mathrm{x}$ is the number of F-T cycles, $\mathrm{t}_{1}$ and $\mathrm{t}_{2}$ are related expressions of the F-T temperature, $\mathrm{t}_{1}=0.0142 \mathrm{~T}^{2}+0.01932 \mathrm{~T}+1.1712, \mathrm{t}_{2}=-0.00054 \mathrm{~T}^{2}-$ $0.00219 \mathrm{~T}+0.02342$ and $\mathrm{T}$ is $\mathrm{F}-\mathrm{T}$ temperature.

The porosity expression in formula (8) is substituted into formula (4) to obtain a permeability model considering temperature changes and cycles:

$$
\mathrm{K}=4.32 \times 10^{-7}\left(\mathrm{t}_{1} \cdot \exp ^{\mathrm{t}_{2} \cdot x}\right)^{1.0642} \times S_{\mathrm{c}} \times T_{2 g}^{2.1632}
$$




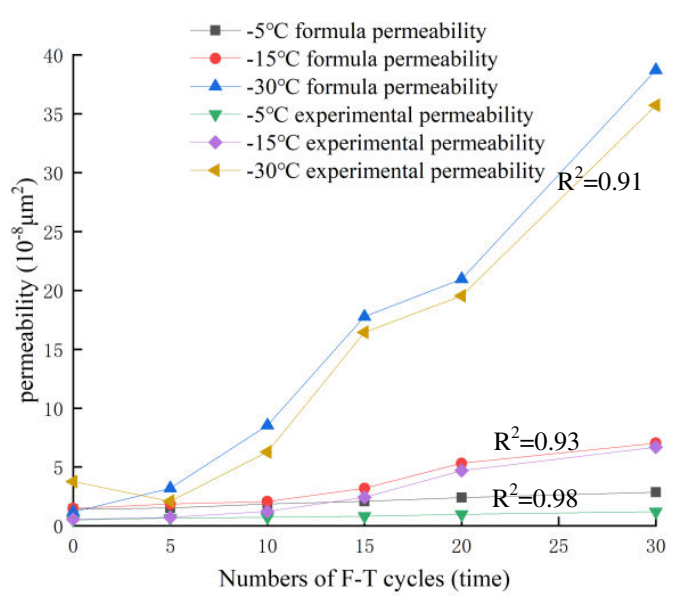

Figure 8 Comparison of experimental and calculated permeability

The theoretical permeability of the F-T shale was calculated by model formula (8), and the influence of freezing temperature is not as significant as the number of cycles. The calculated permeability model modified by Sc is compared with the experimental value, as shown in Figure 8.The fitting correlation coefficient of Fig. 8 is greater than 0.91 , indicating the good fit and verifying the applicability of the modified model.

\section{Conclusion}

We analyze the NMR spectral evolution and permeability experiments of shale under the freeze-melting cycles and study the evolution law of shale internal pore structure and permeability. In addition, then get these conclusions:

At the same freezing temperature, with the increasing number of cycles, the rock sample pore structure changes are mainly manifested by the micro pores, small pore development expansion into medium holes, and the middle hole expansion into large holes, and all kinds of aperture pores are increase. In addition, large pores and cracks should not only be more affected by freezing and melting than micropores, small pores and medium pores but also be affected in advance of micropores, small pores and medium holes. At the same number of cycles, compared with different freezing temperatures, the large holes and cracks were found to be more affected by the temperature.

As the number of F-T cycles increases and the freezing temperature decreases, the average porosity of shale at the three freezing temperatures increases and the increase gradually increases. Compared with pore analysis of different pore sizes, it is found that the change in porosity is caused by the increase in size pore and the continuous increase in pore size. At a freezing temperature of $-15^{\circ} \mathrm{C}$, the shale permeability begins to increase significantly; at a freezing temperature of $-30^{\circ} \mathrm{C}$, the permeability increases by an order of magnitude. Through comparison, it is found that the increase of shale porosity and permeability shows a good positive correlation, indicating that the expansion of pore structure and the increase of pore number lead to the increase of porosity, and the increase of porosity further leads to the increase of permeability.

In the study of the permeability model, based on the improved SDR permeability model, Sc was added to modify the model. The introduction of F-T cycles and freezing temperature parameters obtained the F-T shale permeability evolution model. The permeability measurement results were compared with the experimental results, and the correlation coefficient was greater than 0.91 , indicating that the model has good applicability. 


\section{Data availability}

The data used to support the findings of this study are included within the article.

\section{References}

[1]WU Yang. Study on strength, damage and creep characteristics of rock-like materials under freeze-thaw cycles[D]. Qingdao University of Science and Technology, (2018)

[2]FU Yan. Study on water-rock interaction with the cyclic drying-wetting effect on rock[D].University Of Chongqing, (2010)

[3]De Kock Tim,Boone Marijn A et al. A pore-scale study of fracture dynamics in rock using X-ray micro-CT under ambient freeze-thaw cycling.[J]. Environmental science \& technology, 49(5). (2015)

[4]M.Takarli, W. Prince. Permeability and P-wave velocity change in granitic rocks under freeze-thaw cycles[J]. Geomechanics and Geoengineering: An International Journal Vol. 2, No. 3 , September, 227--234. (2007)

[5]Zhang, C., Tang, J. X., Teng, J. Y. \& Li, C. L. Experimental study of infuences of pore number and pore size on mechanical properties of marble. Rock Soil Mech. 38(S2), 41-50. (2017).

[6]Hiroaki Izumiyama, Daizo Tsutsumi, Masaharu Fujita. Effect of freeze-thaw action on porosity change and destruction of weathered bedrock in different lithology and development of destruction model[J]. International Journal of Japan Erosion Control Engineering, Vol. 5, No. 1, (2012)

[7]Muhammad Shahzad Kamal et al. Clay minerals damage quantification in sandstone rocks using core flooding and NMR[J]. Journal of Petroleum Exploration and Production Technology, 9(1). (2019)

[8]Wang, Q. Z., Yang, J. R., Zhang, C. G. \& Zhou, Y. Determination of dynamic crack initiation and propagation toughness of a rock using a hybrid experimental-numerical approach. J. Eng. Mech. 142(12), 04016097. (2016)

[9]Coralie Genty, Jerry L. Jensen, Wayne M. Ahr. Distinguishing Carbonate Reservoir Pore Facies with Nuclear Magnetic Resonance Measurements[J]. Natural Resources Research, 16(1). (2007)

[10]Zhu Zhende, HUANG Qiang et al. Mesoscopic experiment on degradation evolution of rock deformation and its meso-damage mechanical model[J]. Journal of Rock Mechanics and Engineering, 32(06):1167-1175. (2013)

[11]LI Jie-lin, ZHU Long-yin, ZHOU Ke-ping et al. Damage characteristics of sandstone pore structure under freeze-thaw cycles[J].Rock and Soil Mechanics, 40(09):3524-3532. (2019)

[12]YAO Yanbin, LIU Dameng. Petrophysical properties and fluids transportation in gas shale: A NMR relaxation spectrum analysis method[J]. Journal of China Coal Society, 43(01):181-189. (2018)

[13]LI Jielin. Experimental study on the deterioration mechanism of rock under the conditions of freezing-thawing cycles in cold regions based on NMR technology[D]. Central South University, (2012)

[14]Meng Fandong, Zhai Yue et al. Experimental study on dynamic tensile properties and energy evolution of sandstone after freeze-thaw cycles[J/OL]. Chinese Journal of Rock Mechanics and Engineering. doi:10.13722/. (2021) 
[15]Hao, X. J., Yuan, L. A. \& Zhao, Y. X. Infuence of initial microcrack on the physic-mechanical properties of rock with slaty cleavage. Geotech. Geol. Eng. 35(5), 2351-2360. (2017)

[16]LI Jielin, LIU Hanwen, Establishment and analysis of the volumetric deformation model of sandstone pores under the effect of freezing-thawing cycles[J]. Journal of Glaciology and Geocryology, 40(06):1173-1180. (2018)

[17]YU Jin, ZHANG Xin, CAI Yan-yan et al. Meso-damage and mechanical properties degradation of sandstone under combined effect of water chemical corrosion and freeze-thaw cycles[J]. Rock and Soil Mechanics, 40(02): 455-464. (2019)

[18]ZHU Linqi. Study on evaluation of pore strucyure and permeability of tight sandstone reservoir by nuclear magnetic resonance logging[D]. Changjiang University, (2017)

[19]BAI Songtao, Cheng Daojie Wan Jinbin et al.Quantitative characterization of sandstone NMR T2 spectrum[J]. Acta Petrolei Sinica, 37(03): 382-391+414. (2016)

[20]XI Baoping, CHENG Zepeng et al . Experimental study on permeability characteristics and its evolution of granite after high temperature. Chinese Journal of Rock Mechanics and Engineering. 40.S1(2021):2716-2723. doi:10.13722/j.cnki.jrme.1026. (2020)

[21]Sun Huanquan, Wang Haitao,and Lun Zengmin. "Effect of permeability and fractures on oil mobilization of unconventional resources during $\mathrm{CO} 2 \mathrm{EOR}$ using nuclear magnetic resonance" Scientific reports 11.1: doi:10.1038/S41598-021-92158-3. (2021)

[22]ZHAI Cheng, Sun Yong. Experimental study on evolution of pore structure in coal after cyclic cryogenic fracturing[J]. Coal Science and Technology,45(06):24-29. (2017)

[23]QIN Lei. Pore evolution after fracturing with cycle liquid nitrogen and the mechanism of permeability enhancement. [D]. China Mining University. (2018)

[24]SHEN Yanjun YANG Gengshe, WANG Ting et al. Evaluation of frost heave force models of pore/fissure in rock and their applicability[J]. Journal of Glaciology and Geocryology, 41(01):117-128. (2019)

[25]SHEN Yanjun YANG Gengshe, RONG Tenglong et al. Localized damage effects of quasi-sandstone with single fracture and fracture behaviors of joint end under cyclic freezing and thawing[J]. Chinese Journal of Rock Mechanics and Engineering, 36(03): 562-570. (2017)

[26]JIA Hailiang XIANG Wei, SHEN Yanjun et al. Discussion of the key issues within calculation of the fatigue damage of rocks subjected to freeze-thaw cycles[J]. Chinese Journal of Rock Mechanics and Engineering, 36(02): 335-346. (2017)

[27]Wu Xing-hui LI Peng et al. Research progress on the evolution of physical and mechanical properties of thermally damaged rock [J/OL]Chinese Journal of Engineering doi: . 1-1610.1297.TF.20210827.1227.002. (2021)

[28]Didem Eren Sarici, Engin Ozdemir. Determining point load strength loss from porosity, Schmidt hardness, and weight of some sedimentary rocks under freeze-thaw conditions[J]. Environmental Earth Sciences 77: 62. (2018)

[29]Sun Huanquan, Wang Haitao, and Lun Zengmin. "Effect of permeability and fractures on oil mobilization of unconventional resources during $\mathrm{CO} 2 \mathrm{EOR}$ using nuclear magnetic resonance" Scientific reports 11.1: doi:10.1038/S41598-021-92158-3. (2021)

[30]XI Baoping, CHENG Zepeng et al. Experimental study on permeability characteristics and its evolution of granite after high temperature.Chinese Journal of Rock Mechanics and Engineering. 40.S1(2021):2716-2723. doi:10.13722/j.cnki.jrme.1026. (2020)

[31]ZHOU Shangwen, XUE Huaqing, GUO Wei et al. A new nuclear magnetic resonance 
permeability model of shale of long maxi formation in southern Sichuan Basin[J]. Journal of China University of Petroleum(Edition of Natural Science), 40(01):56-61. (2016)

\section{Acknowledgments}

This work was supported by the National Natural Science Foundation of China (2016YFC0600704), and the School of Mechanics of Liaoning University of Engineering and Technology for its support.

\section{Author contributions}

Professor Wang proposed the main idea and scheme of the experiment. Xuan was in charge of the experiment, and both wrote the paper at the same time. The remaining authors examined the accuracy of the article and suggested some modifications. All authors reviewed the manuscript.

\section{Competing interests}

Te authors declare no competing interests. 